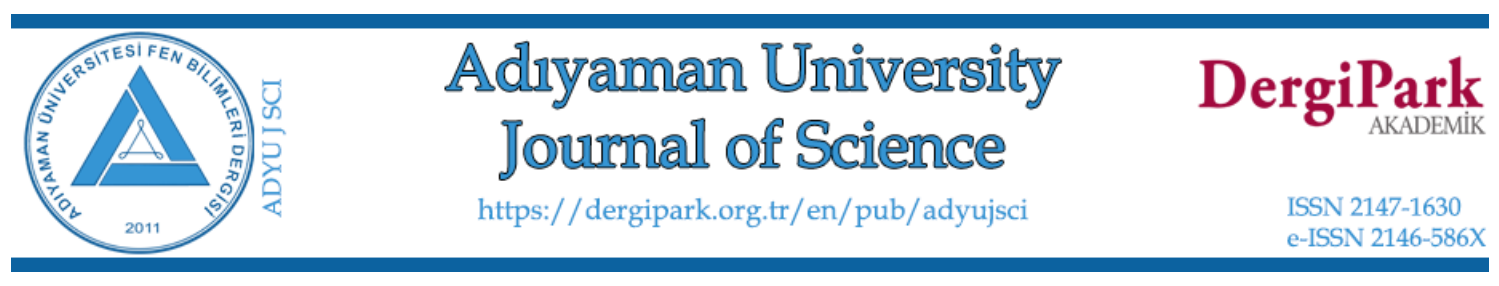

\title{
On the Harmonic Evolute Surfaces of Hasimoto Surfaces
}

\author{
Kemal EREN ${ }^{1, *}$, Alev KELLECİ AKBAY ${ }^{2}$

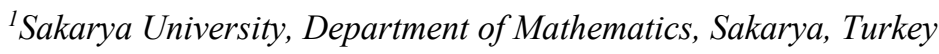 \\ kemal.eren1@ogr.sakarya.edu.tr,ORCID:0000-0001-5273-7897 \\ ${ }^{2}$ Firat University, Department of Mathematics, Elazığ, Turkey \\ alevkelleci@hotmail.com,ORCID:0000-0003-2528-2131
}

\begin{tabular}{lll}
\hline Received: 03.11 .2020 & Accepted: 30.04 .2021 & Published: 30.06 .2021
\end{tabular}

\begin{abstract}
In this study, firstly by considering the evolution of a moving space curve, we give some related definitions and some new results about Hasimoto surfaces in Euclidean 3-spaces. Secondly, we examine harmonic evolute surfaces of Hasimoto surfaces in Euclidean 3-spaces and also, we give some geometric properties of these type surfaces. Moreover, we express the properties of parameter curves of harmonic evolute surfaces in Euclidean space. Finally, we give an explicit example of Hasimoto surface and its harmonic evolute surface and also we plot these surfaces.
\end{abstract}

Keywords: Hasimoto surfaces; Harmonic evolute surface; Binormal motion; Evolution of curves and surfaces; Gaussian curvature; Mean curvature.

\section{Hasimoto Yüzeylerin Harmonik Evrim Yüzeyleri Üzerine}

$\ddot{O} \mathbf{z}$

Bu çalısmada, ilk olarak hareketli bir uzay eğrisinin evrimini ele alarak, Öklid 3uzaylarında Hasimoto yüzeyleri ile ilgili bazı tanımlar ve yeni sonuçlar verilmiştir. İkinci olarak, Öklid 3-uzaylarında Hasimoto yüzeylerinin harmonik evrim yüzeyleri incelenmiştir ve ayrıca bu tip yüzeylerin bazı geometrik özellikleri verilmiştir. Ayrıca, Öklid uzayındaki harmonik evrim 
yüzeylerinin parametre eğrilerinin özelliklerini ifade edilmiştir. Son olarak Hasimoto yüzeyinin ve harmonik evrimsel yüzeyinin açık bir örneği verilmiş ve ayrıca bu yüzeyler çizilmiştir.

Anahtar Kelimeler: Hasimoto yüzeyler; Harmonik evrim yüzeyler; Binormal hareketi; Eğri ve yüzeylerin evrimi; Gaussian eğrilik; Ortalama eğrilik.

\section{Introduction}

The harmonic evolute surface of a given surface $r$ is defined as the geometric location of points that are as far away as the opposite of the mean curvature from the surface in the direction of the normal of the surface. The harmonic surface of a surface must be non-minimal in order to be obtained. Let $r=r(s, t)$ be a surface and also $u$, its normal and $H$ denotes its non-zero mean curvature. Then, the harmonic evolute surface of $r=r(s, t)$ is given by parametrically

$$
h(s, t)=r(s, t)+\frac{1}{H(s, t)} u(s, t),
$$

see for more details, [1-3].

In recent years, the theory of surfaces with the connection of the motion of space curves and differential equations is a subject of research attention, [4, 5]. At the same time, the applications of this subject in differential geometry and physics have attracted attention. In 1971, Hasimoto examined the movement of a vortex filament, and then in 1972, Hasimoto showed that vortex filament (smoke ring) equation is equivalent non-linear Schrodinger equation $[6,7]$. The relationship between the integrable equations and the theory of surfaces produces new types of surfaces. Hasimoto surfaces are one of these surfaces. Let $r=r(s, t)$ be the position vector of a moving curve on a surface in Euclidean 3-space and for all $t$-parameter, $r=r(s, t)$ be a unit speed curve. If this surface is a Hasimoto surface, then, the position vector $r=r(s, t)$ satisfies the following condition

$$
r_{t}=r_{s} \wedge r_{s s}
$$

where $t$ is the time parameter, $s$ is arc-length parameter and the subscripts indicate the partial differential. This equation is said to be smoke ring equation or the vortex filament. The geometric properties of Hasimoto surfaces are investigated in detail by [8,9]. Hashimoto surfaces have been produced according to the Bishop frame in Euclidean space, see [10]; and in Minkowski space, see [11]. Also, parallel surfaces of Hasimoto surfaces in Euclidean space are investigated at [12]. 
The aim of this study is to investigate the geometric properties the harmonic evolute surfaces of Hasimoto surfaces. Also, the characterization of the $s$ and $t$ parameter curves of the harmonic evolute surfaces of the Hasimoto surface is examined.

\section{Geometric Preliminaries}

In Euclidean 3-space, Euclidean inner product is given by $\langle x, y\rangle=x_{1} y_{1}+x_{2} y_{2}+x_{3} y_{3}$ for two vectors $x=\left(x_{1}, x_{2}, x_{3}\right)$ and $y=\left(y_{1}, y_{2}, y_{3}\right)$ in $E^{3}$. The norm of a given vector $x \in E^{3}$ is

defined as $\|x\|=\sqrt{\langle x, x>}$. Also, for an arbitrary curve $r$ in $E^{3}$, if $\left\|r^{\prime}(s)\right\|=1$, then the curve $r$ is called as a unit speed curve or parametrized by arc-length parameter $s$ in Euclidean 3-space, $E^{3}$.

Now, we summarize basic concepts about the evolution of a moving space curve in Euclidean 3-space and so we give the intrinsic equations expressing with curvatures of it corresponding with moving Frenet frame $\{T, N, B\}$, [8]. Let $r$ be a moving space curve described in parametric form by a position vector $r(s, t)$ in Euclidean 3-space, where $s$ is the arc-length parameter and $t$ is the time parameter. Thus, the moving Frenet frame $\{T, N, B\}$ of the curve with respect to $s$ and $t$ can be written in matrix form as follows:

$$
\left[\begin{array}{l}
T \\
N \\
B
\end{array}\right]_{s}=\left[\begin{array}{ccc}
0 & \kappa & 0 \\
-\kappa & 0 & \tau \\
0 & -\tau & 0
\end{array}\right]\left[\begin{array}{l}
T \\
N \\
B
\end{array}\right] \text { and }\left[\begin{array}{c}
T \\
N \\
B
\end{array}\right]_{t}=\left[\begin{array}{ccc}
0 & \alpha & \beta \\
-\alpha & 0 & \gamma \\
-\beta & -\gamma & 0
\end{array}\right]\left[\begin{array}{c}
T \\
N \\
B
\end{array}\right] .
$$

Here $\alpha, \beta$, and $\gamma$ are some smooth functions of $s$ and $t$. Moreover, the time evolution equation for the curvature $\kappa$ and the torsion $\tau$ of a moving curve can be found as

$$
\begin{aligned}
& \kappa_{t}=\alpha_{s}-\tau \beta, \\
& \tau_{t}=\gamma_{s}+\kappa \beta,
\end{aligned}
$$

where

$$
\gamma=\frac{\beta_{s}+\tau \alpha}{\kappa}
$$

\subsection{Some well-known and new results for Hasimoto surfaces}

In this section, firstly we mention about some known geometric properties of $r=r(s, t)$ Hasimoto surfaces (or called as NLS surfaces) in Euclidean 3-spaces swept out by the binormal motion $r_{t}=\kappa B$, (see for more details, [7-9]). As well known that, since the tangent vector of 
$r(s, t)$ Hasimoto surfaces is $r_{s}=T$, so the unit normal vector field $u$ of this surface is calculated by [9]

$$
u=\frac{r_{s} \wedge r_{t}}{\left\|r_{s} \wedge r_{t}\right\|}=-N
$$

Moreover, the coefficients of the first and second fundamental form of the Hasimoto surfaces are obtained by [9], respectively,

$$
\begin{aligned}
& E=\left\langle r_{s}, r_{s}\right\rangle=1, F=\left\langle r_{s}, r_{t}\right\rangle=0, G=\left\langle r_{t}, r_{t}\right\rangle=\kappa^{2}, \\
& e=\left\langle r_{s s}, u\right\rangle=-\kappa, f=\left\langle r_{s t}, u\right\rangle=\kappa \tau, g=\left\langle r_{t t}, u\right\rangle=\kappa_{s s}-\kappa \tau^{2} .
\end{aligned}
$$

Moreover, by using the compatibility conditions, $T_{s t}=T_{t s}, N_{s t}=N_{t s}, B_{s t}=B_{t s}$ and Eqn. (2), one can easily get

$$
\alpha=-\kappa \tau, \beta=\kappa_{s}, \gamma=\frac{\kappa_{s s}-\kappa \tau^{2}}{\kappa},
$$

where these smooth functions are defined as in Eqn. (2). So, the intrinsic equations given as in Eqn. (3) for a moving curve can be rewritten as

$$
\begin{aligned}
& \kappa_{t}=-2 \kappa_{s} \tau-\kappa \tau_{s}, \\
& \tau_{t}=\kappa \kappa_{s}-2 \tau \tau_{s}+\left(\frac{\kappa_{s s}}{\kappa}\right)_{s} .
\end{aligned}
$$

In order to study the harmonic evolute surfaces of Hasimoto surfaces, first let's recall the Gaussian and the mean curvatues of these surfaces in terms of the cuvature and torsion of moving curve:

Corollary 1. [9] Let $r=r(s, t)$ be a Hasimoto surface in Euclidean 3-space. Then its the Gaussian curvature $K$ and the mean the curvature $H$ are given by

$$
K=\frac{e g-f^{2}}{E G-F^{2}}=\frac{-\kappa_{s s}}{\kappa}
$$

and

$$
H=\frac{1}{2} \frac{E g-2 F f+G e}{E G-F^{2}}=\frac{1}{2 \kappa}\left(\frac{\kappa_{s s}}{\kappa}-\kappa^{2}-\tau^{2}\right),
$$

where $\kappa \neq 0$ and $\tau$ are the curvature and torsion of moving curve respectively.

Secondly, we would like to give some characterizations of parametric curves of Hasimoto surfaces in Euclidean 3-spaces. 
Theorem 1. Let $r=r(s, t)$ be a Hasimoto surface in Euclidean 3-spaces such that $r=r(s, t)$ is a curve parametrized by arc-length for all $t$. Then s-parameter curves of the Hasimoto surface are

- geodesic,

- asymptotic if and only if $\kappa=0$.

Proof. Assume that $r=r(s, t)$ is a Hasimoto surface in $E^{3}$. Thus by considering the tangent vector of $r$, we have $r_{s s}=T_{s}=\kappa N$. So the binormal component of $r_{s s}$ is zero. From which, we conclude that $s$-parameter curves of the Hasimoto surfaces are geodesics.

On the other hand, as we know that $r_{s s}=\kappa N$, the normal component of $r_{s s}$ is zero if and only if $\kappa=0$ which shows that $s$-parameter curves of the Hasimoto surfaces are asymptotic ones if and only if $\kappa=0$.

Theorem 2. Let $r=r(s, t)$ be a Hasimoto surface in Euclidean 3-spaces such that $r=r(s, t)$ is a curve parametrized by arc-length for all $t$. Then $t$-parameter curves of $r$ are

- $\quad$ geodesic if and only if $2 \kappa_{s} \tau+\kappa \tau_{s}=0$,

- asymptotic if and only if $\frac{\kappa_{s s}}{\kappa}=\tau^{2}$.

Proof. The proof can be exactly done by similar way of the proof of previous Theorem.

Theorem 3. Let $r=r(s, t)$ be a Hasimoto surface in Euclidean 3-spaces such that $r=r(s, t)$ is a curve parametrized by arc-length for all $t$. Then, parameter curves of $r$ are lines of curvature if and only if. $\kappa \tau=0$.

Proof. From Eqn. (6), we have $F=0$ and $f=\kappa \tau$. Thus we conclude that these curves of the Hasimoto surfaces are lines of curvature if and only if $\kappa \tau=0$.

\section{Harmonic Evolute Surfaces of Hasimoto Surfaces}

In this section, inspired by data from Section 2, we aimed to investigate the harmonic evolute surfaces of the Hasimoto surfaces whose mean curvature does not vanish. Let us assume that the Hasimoto surface $r=r(s, t)$ is not a minimal surface. Then we have harmonic evolute surface of the Hasimoto surface defined as 


$$
h(s, t)=r(s, t)+\frac{1}{H(s, t)} u(s, t)
$$

where $H(s, t)=\frac{1}{2 \kappa}\left(\frac{\kappa_{s s}}{\kappa}-\kappa^{2}-\tau^{2}\right)$ with $\kappa \neq 0$ and $u(s, t)=-N(s, t)$ are the mean curvature and the normal vector of Hasimoto surface, respectively. By considering Eqn. (2) and Eqn. (10) with Eqn. (11), we have

$$
\begin{aligned}
& h_{s}(s, t)=\left(1+\frac{\kappa}{H}\right) T-\left(\frac{1}{H}\right)_{s} N-\frac{\tau}{H} B, \\
& h_{t}(s, t)=\frac{\alpha}{H} T-\left(\frac{1}{H}\right)_{t} N+\left(\kappa-\frac{\gamma}{H}\right) B,
\end{aligned}
$$

where $\alpha, \gamma$ are given in Eqn. (7). So, the normal vector field of the harmonic evolute surface is found as

$$
u^{*}=\frac{h_{s} \wedge h_{t}}{\left\|h_{s} \wedge h_{t}\right\|}=\frac{\lambda_{1} T+\lambda_{2} N+\lambda_{3} B}{\sqrt{\lambda_{1}^{2}+\lambda_{2}^{2}+\lambda_{3}^{2}}}
$$

such that

$$
\begin{aligned}
& \lambda_{1}=-\frac{\tau}{H}\left(\frac{1}{H}\right)_{t}-\left(\frac{1}{H}\right)_{s}\left(\kappa-\frac{\gamma}{H}\right), \\
& \lambda_{2}=-\frac{\tau \alpha}{H^{2}}-\left(1+\frac{\kappa}{H}\right)\left(\kappa-\frac{\gamma}{H}\right), \\
& \lambda_{3}=\frac{\alpha}{H}\left(\frac{1}{H}\right)_{s}-\left(1+\frac{\kappa}{H}\right)\left(\frac{1}{H}\right)_{t}
\end{aligned}
$$

Moreover, the coefficients first fundamental form of the harmonic evolute surface, $h(s, t)$ in Euclidean 3-space are found by

$$
\begin{aligned}
& E^{*}=\left\langle h_{s}, h_{s}\right\rangle=\left(1+\frac{\kappa}{H}\right)^{2}+\left(\left(\frac{1}{H}\right)_{s}\right)^{2}+\left(\frac{\tau}{H}\right)^{2} \\
& F^{*}=\left\langle h_{s}, h_{t}\right\rangle=\frac{\alpha}{H}\left(1+\frac{\kappa}{H}\right)+\left(\frac{1}{H}\right)_{s}\left(\frac{1}{H}\right)_{t}-\frac{\tau}{H}\left(\kappa-\frac{\gamma}{H}\right), \\
& G^{*}=\left\langle h_{t}, h_{t}\right\rangle=\left(\frac{\alpha}{H}\right)^{2}+\left(\left(\frac{1}{H}\right)_{t}\right)^{2}+\left(\kappa-\frac{\gamma}{H}\right)^{2} .
\end{aligned}
$$

On the other hand, the second order derivative formulas of the harmonic evolute surface defined in Eqn. (11) are 


$$
\begin{aligned}
h_{s s} & =\left(\left(1+\frac{\kappa}{H}\right)_{s}+\kappa\left(\frac{1}{H}\right)_{s s}\right) T+\left(\kappa\left(1+\frac{\kappa}{H}\right)-\left(\frac{1}{H}\right)_{s s}+\frac{\tau^{2}}{H}\right) N-\left(\tau\left(\frac{1}{H}\right)_{s s}+\left(\frac{\tau}{H}\right)\right) B, \\
h_{s t}= & \left(\left(1+\frac{\kappa}{H}\right)_{t}+\alpha\left(\frac{1}{H}\right)_{s}+\frac{\beta \tau}{H}\right) T+\left(\alpha\left(1+\frac{\kappa}{H}\right)-\left(\frac{1}{H}\right)_{s t}+\frac{\gamma \tau}{H}\right) N \\
& +\left(\beta\left(1+\frac{\kappa}{H}\right)-\gamma\left(\frac{1}{H}\right)_{s}-\left(\frac{\tau}{H}\right)_{t}\right) B, \\
h_{t t}= & \left(\left(\frac{\alpha}{H}\right)_{t}+\alpha\left(\frac{1}{H}\right)_{t}-\left(\kappa-\frac{\gamma}{H}\right)\right)_{t} T+\left(\frac{\alpha^{2}}{H}-\left(\frac{1}{H}\right)_{t t}-\gamma\left(\kappa-\frac{\gamma}{H}\right)\right) N \\
& +\left(\frac{\alpha \beta}{H}-\gamma\left(\frac{1}{H}\right)_{t}+\left(\kappa-\frac{\gamma}{H}\right)\right) B .
\end{aligned}
$$

By considering last equations with Eqn. (12), we could find the coefficients of second fundamental form of the harmonic evolute surface $h(s, t)$ as

$$
\begin{aligned}
& e^{*}=\frac{\lambda_{1}\left(\left(1+\frac{\kappa}{H}\right)_{s}+\kappa\left(\frac{1}{H}\right)_{s s}\right)+\lambda_{2}\left(\kappa\left(1+\frac{\kappa}{H}\right)-\left(\frac{1}{H}\right)_{s s}+\frac{\tau^{2}}{H}\right)-\lambda_{3}\left(\tau\left(\frac{1}{H}\right)_{s s}+\left(\frac{\tau}{H}\right)_{s}\right)}{\sqrt{\lambda_{1}^{2}+\lambda_{2}^{2}+\lambda_{3}^{2}}}, \\
& f^{*}=\frac{\lambda_{1}\left(\left(1+\frac{\kappa}{H}\right)_{t}+\alpha\left(\frac{1}{H}\right)_{s}+\frac{\beta \tau}{H}\right)+\lambda_{2}\left(\alpha\left(1+\frac{\kappa}{H}\right)-\left(\frac{1}{H}\right)_{s t}+\frac{\gamma \tau}{H}\right)+\lambda_{3}\left(\beta\left(1+\frac{\kappa}{H}\right)-\gamma\left(\frac{1}{H}\right)-\left(\frac{\tau}{H}\right)\right)}{\sqrt{\lambda_{1}^{2}+\lambda_{2}^{2}+\lambda_{3}^{2}}}, \\
& g^{*}=\frac{\lambda_{1}\left(\left(\frac{\alpha}{H}\right)_{t}+\alpha\left(\frac{1}{H}\right)_{t}-\left(\kappa-\frac{\gamma}{H}\right)\right)+\lambda_{2}\left(\frac{\alpha^{2}}{H}-\left(\frac{1}{H}\right)_{u}-\gamma\left(\kappa-\frac{\gamma}{H}\right)\right)+\lambda_{3}\left(\frac{\alpha \beta}{H}-\gamma\left(\frac{1}{H}\right)_{t}+\left(\kappa-\frac{\gamma}{H}\right)\right)}{\sqrt{\lambda_{1}^{2}+\lambda_{2}^{2}+\lambda_{3}^{2}}} .
\end{aligned}
$$

From the equations Eqn. (13) and Eqn. (15), the Gaussian curvature $K^{*}$ and the mean curvature $H^{*}$ of the harmonic evolute surface can be obtained as following 


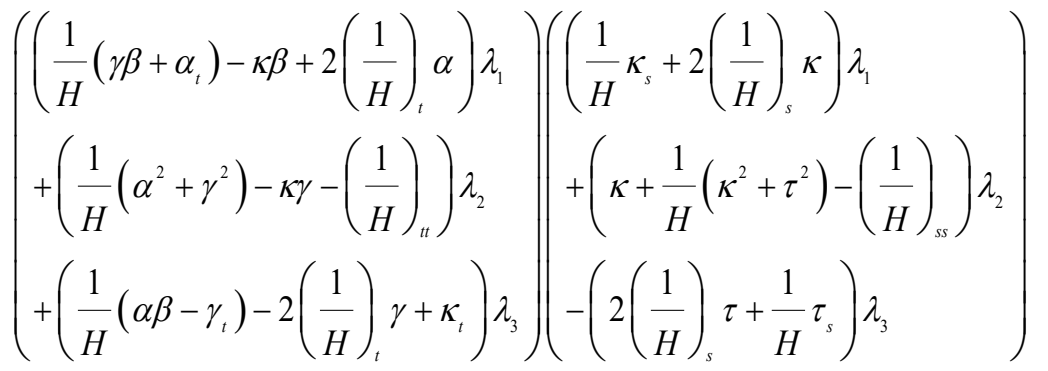

$$
\begin{aligned}
& K^{*}=\frac{\left(\left(\begin{array}{l}
\left.\left.\left(\frac{1}{H}\right)_{s} \alpha+\left(\frac{1}{H}\right)_{t} \kappa+\frac{1}{H}\left(\beta \tau+\kappa_{t}\right)\right) \lambda_{1}\right)^{2} \\
+\left(\alpha n+\frac{1}{H}(\gamma \tau+\kappa \alpha)-\left(\frac{1}{H}\right)_{s t}\right)_{2} \lambda_{2} \\
\left.+\left(\beta+\frac{1}{H}\left(\kappa \beta-\tau_{t}\right)-\gamma H_{s}-\tau H_{t}\right) \lambda_{3}\right)^{2}
\end{array}\right.\right.}{\left(\begin{array}{l}
-\left(\frac{1}{H}\left(\alpha+\frac{1}{H}(\kappa \alpha+\gamma \tau)-\kappa \tau\right)+\left(\frac{1}{H}\right)\left(\frac{1}{H}\right)_{t}\right)^{2} \\
+\left(\left(1+\frac{1}{H} \kappa\right)^{2}+\frac{1}{H^{2}} \tau^{2}+\left(\frac{1}{H}\right)_{s}^{2}\right)\left(\frac{1}{H^{2}} \alpha^{2}+\left(-\frac{1}{H} \gamma+\kappa\right)^{2}+\left(\frac{1}{H}\right)_{t}^{2}\right)
\end{array}\right)\left(\lambda_{1}^{2}+\lambda_{2}^{2}+\lambda_{3}^{2}\right)}
\end{aligned}
$$

and 


$$
\begin{aligned}
& \left(\left(1+\frac{\kappa}{H}\right)^{2}+\left(\frac{\tau}{H}\right)^{2}+\left(\frac{1}{H}\right)_{s}^{2}\right)\left(\begin{array}{l}
\left(\frac{\gamma \beta+\alpha_{t}}{H}-\kappa \beta+2\left(\frac{1}{H}\right)_{t} \alpha\right) \lambda_{1} \\
+\left(\frac{\alpha^{2}+\gamma^{2}}{H}-\kappa \gamma-\left(\frac{1}{H}\right)_{t t}\right) \lambda_{2} \\
+\left(\frac{\alpha \beta-\gamma_{t}}{H}-2 \gamma\left(\frac{1}{H}\right)_{t}+\kappa_{t}\right) \lambda_{3}
\end{array}\right) \\
& +\left(\left(\frac{\alpha}{H}\right)^{2}+\left(-\frac{\gamma}{H}+\kappa\right)^{2}+\left(\frac{1}{H}\right)_{t}^{2}\right)\left(\begin{array}{l}
\left(\frac{1}{H} \kappa_{s}+2 \kappa\left(\frac{1}{H}\right)_{s}\right) \lambda_{1} \\
+\left(\kappa+\frac{\kappa^{2}+\tau^{2}}{H}-\left(\frac{1}{H}\right)_{s s}\right) \lambda_{2} \\
-\left(2 \tau\left(\frac{1}{H}\right)_{s}+\frac{\tau_{s}}{H}\right) \lambda_{3}
\end{array}\right) \\
& \left(\frac{1}{H}\left(\alpha+\frac{\kappa \alpha+\gamma \tau}{H}-\kappa \tau\right)\right)\left(\left(\alpha\left(\frac{1}{H}\right)_{s}+\kappa\left(\frac{1}{H}\right)_{t}+\frac{\beta \tau+\kappa_{t}}{H}\right) \lambda_{1}\right. \\
& -2\left(\begin{array}{l}
\frac{1}{H}\left(\alpha+\frac{\kappa \alpha+\gamma \tau}{H}-\kappa \tau\right) \\
+\left(\frac{1}{H}\right)_{s}\left(\frac{1}{H}\right)
\end{array}\right)+\left(\alpha n+\frac{\gamma \tau+\kappa \alpha}{H}-\left(\frac{1}{H}\right)_{s t}\right) \lambda_{2} \\
& H^{*}=\frac{2\left(-\left(\frac{1}{H}\left(\alpha+\frac{\kappa \alpha+\gamma \tau}{H}-\kappa \tau\right)+\left(\frac{1}{H}\right)_{s}\left(\frac{1}{H}\right)_{t}\right)^{2}\right.}{\left(+\left(\left(1+\frac{\kappa}{H}\right)^{2}+\left(\frac{\tau}{H}\right)^{2}+\left(\frac{1}{H}\right)_{s}^{2}\right)\left(\left(\frac{\alpha}{H}\right)^{2}+\left(\kappa-\frac{\gamma}{H}\right)^{2}+\left(\frac{1}{H}\right)_{t}^{2}\right)\right) \sqrt{\lambda_{1}^{2}+\lambda_{2}^{2}+\lambda_{3}^{2}}} .
\end{aligned}
$$

Corollary 2. The harmonic evolute surface $h(s, t)$ of the Hasimoto surface $r(s, t)$ is neither a developed surface nor a minimal surface.

Let's give some theorems about geometric interpretation of parametric curves of the harmonic evolute surface of the Hasimoto surface.

Theorem 4. If we assume that $h(s, t)$ is a harmonic evolute surface of the Hasimoto surface $r(s, t)$ in Euclidean 3-space, then $s$-parameter curves of $h(s, t)$ are

- geodesic if and only if $\tau\left(\frac{1}{H}\right)_{s s}+\left(\frac{\tau}{H}\right)_{s}=0$, 
- asymptotic if and only if $\kappa\left(1+\frac{\kappa}{H}\right)-\left(\frac{1}{H}\right)_{s s}+\frac{\tau^{2}}{H}=0$.

Proof. Let us assume that $h=h(s, t)$ is a harmonic evolute surface of a Hasimoto surface in Euclidean 3-space. Then by considering the first expression in Eqn. (14), we have the binormal component of $h_{s s}$ is $\tau\left(\frac{1}{H}\right)_{s s}+\left(\frac{\tau}{H}\right)_{s}$. From which, we conclude that $s$-parameter curves of the harmonic evolute surfaces are geodesics if and only if $\tau\left(\frac{1}{H}\right)_{s s}+\left(\frac{\tau}{H}\right)_{s}=0$.

On the other hand, by considering the first expression in Eqn. (14), we have the normal component of $h_{s s}$ is $\kappa\left(1+\frac{\kappa}{H}\right)-\left(\frac{1}{H}\right)_{s s}+\frac{\tau^{2}}{H}$, which means that $s$-parameter curves of the Hasimoto surfaces are asymptotics if and only if $\kappa\left(1+\frac{\kappa}{H}\right)-\left(\frac{1}{H}\right)_{s s}+\frac{\tau^{2}}{H}=0$.

Theorem 5. If we assume that $h(s, t)$ is a harmonic evolute surface of the Hasimoto surface $r(s, t)$ in Euclidean 3-space, then t-parameter curves of $h(s, t)$ are

- geodesic if and only if $\frac{\alpha \beta}{H}-\gamma\left(\frac{1}{H}\right)_{t}+\left(\kappa-\frac{\gamma}{H}\right)_{t}=0$

- asymptotic if and only if $\frac{\alpha^{2}}{H}-\left(\frac{1}{H}\right)_{t t}-\gamma\left(\kappa-\frac{\gamma}{H}\right)=0$,

where $\alpha=-\kappa \tau, \beta=\kappa_{s}$ and $\gamma=\frac{\kappa_{s s}-\kappa \tau^{2}}{\kappa}$ are defined as in Eqn. (8).

Proof. Let us assume that $h=h(s, t)$ is a harmonic evolute surface of a Hasimoto surface in Euclidean 3-space. Then by considering the last expression in Eqn. (14), we have the binormal component of $h_{t t}$ is $\frac{\alpha \beta}{H}-\gamma\left(\frac{1}{H}\right)_{t}+\left(\kappa-\frac{\gamma}{H}\right)_{t}$. From which, we conclude that $s$-parameter curves of the harmonic evolute surfaces are geodesics if and only if $\frac{\alpha \beta}{H}-\gamma\left(\frac{1}{H}\right)_{t}+\left(\kappa-\frac{\gamma}{H}\right)_{t}=0$. 
On the other hand, by considering the last expression in Eqn. (14), we have the normal component of $h_{t t}$ is $\frac{\alpha^{2}}{H}-\left(\frac{1}{H}\right)_{t t}-\gamma\left(\kappa-\frac{\gamma}{H}\right)$, which means that $s$-parameter curves of the Hasimoto surfaces are asymptotics if and only if $\frac{\alpha^{2}}{H}-\left(\frac{1}{H}\right)_{t t}-\gamma\left(\kappa-\frac{\gamma}{H}\right)=0$.

Corollary 3. Let $h(s, t)$ be a harmonic evolute surface of Hasimoto surface $r(s, t)$. Then the following statements are satisfied;

- while there is no conditions for the $s$-parameter curves of the Hasimoto surface to be geodesic, the $s$-parameter curves of the harmonic evolute surfaces are geodesic under the condition is $\tau\left(\frac{1}{H}\right)_{s s}+\left(\frac{\tau}{H}\right)_{s}=0$.

- the $t$-parameter curves of the Hasimoto surfaces are geodesic under the condition that $2 \kappa_{s} \tau+\kappa \tau_{s}=0$, while the $t$-parameter curves of the harmonic evolute surfaces are geodesic under the condition is. $\frac{\alpha \beta}{H}-\gamma\left(\frac{1}{H}\right)_{t}+\left(\kappa-\frac{\gamma}{H}\right)_{t}=0$.

Theorem 9. Let $h(s, t)$ be a harmonic evolute surface of the Hasimoto surface $r(s, t)$. The parameter curves of harmonic evolute surface are lines of curvature if and only if $\tau=0$ and $\kappa$ is a non-zero constant.

Proof. The parameter curves of the harmonic evolute surface $h(s, t)$ are lines of curvature if and only if $F^{*}$ and $f^{*}$ being the coefficients of the first and second fundamental form, respectively, must be vanish. From the equations Eqn. (13) and Eqn. (15), we yield $F^{*}=f^{*}=0$ , if $\tau=0$ and $\kappa$ is a non-zero constant. Thus the proof is completed.

\section{Example}

Now, we give and plot a nice example related with the Hasimoto surface satisfying the Eqn. (1) and also its harmonic evolute surface. For this, if one take $\kappa=2 \operatorname{sech}(s)$ and $\tau=0$ such that they satisfy the Eqn. (8), then the Hasimoto surface $r(s, t)$ (see in Fig. 1) is expressed as follows

$$
r(s, t)=(s-2 \tanh (s),-2 \operatorname{sech}(s) \cos (t),-2 \operatorname{sech}(s) \sin (t)) .
$$




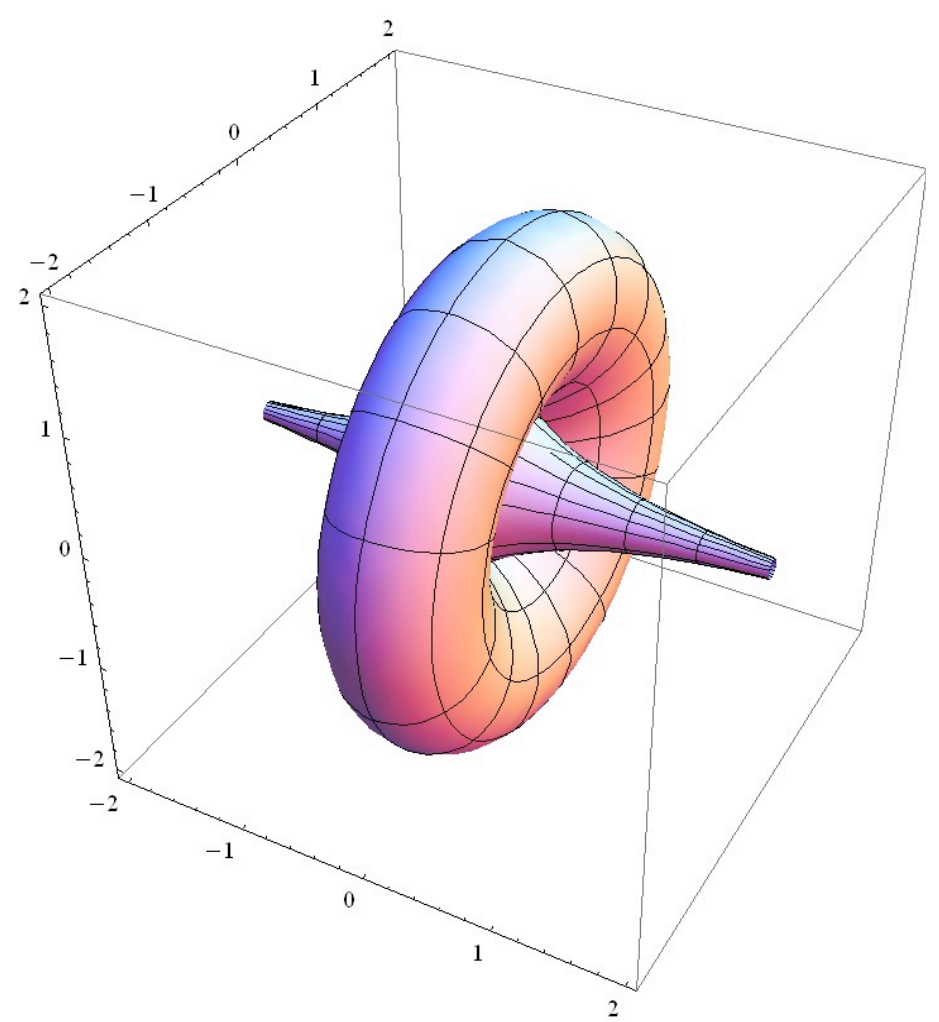

Figure 1: The Hasimoto surface $r(s, t)$ with $s \in(-4,4)$ and $t \in(0,2 \pi)$

So, the normal vector field and the mean curvature of the Hasimoto surface $r$ are given by, respectively,

$$
u=\left(\begin{array}{l}
-2 \sqrt{\operatorname{sech}\left(s^{2}\right)} \tanh (s), \frac{1}{2} \cos (t)(-3+\cosh (2 s)) \operatorname{sech}(s) \sqrt{\operatorname{sech}\left(s^{2}\right)} \\
\frac{1}{2}(-3+\cosh (2 s)) \operatorname{sech}(s) \sqrt{\operatorname{sech}\left(s^{2}\right)} \sin (t)
\end{array}\right)
$$

and

$$
H=\frac{1}{4} \operatorname{sech}(s)\left(-5+\sinh \left(s^{2}\right)\right)
$$

Thus from Eqn. (11), we obtain the parametrization of the harmonic evolute surface $h(s, t)$ (see in Fig. 2) as 


$$
h(s, t)=\left(\begin{array}{c}
s-\frac{8 \sqrt{\operatorname{sech}\left(s^{2}\right)} \sinh (s)}{-5+\sinh \left(s^{2}\right)}-2 \tanh (s), \\
2 \cos (t)\left(-\operatorname{sech}(s)+\frac{(-3+\cosh (2 s)) \sqrt{\operatorname{sech}\left(s^{2}\right)}}{-5+\sinh \left(s^{2}\right)}\right) \\
2 \sin (t)\left(-\operatorname{sech}(s)+\frac{(-3+\cosh (2 s)) \sqrt{\operatorname{sech}\left(s^{2}\right)}}{-5+\sinh \left(s^{2}\right)}\right)
\end{array}\right) .
$$

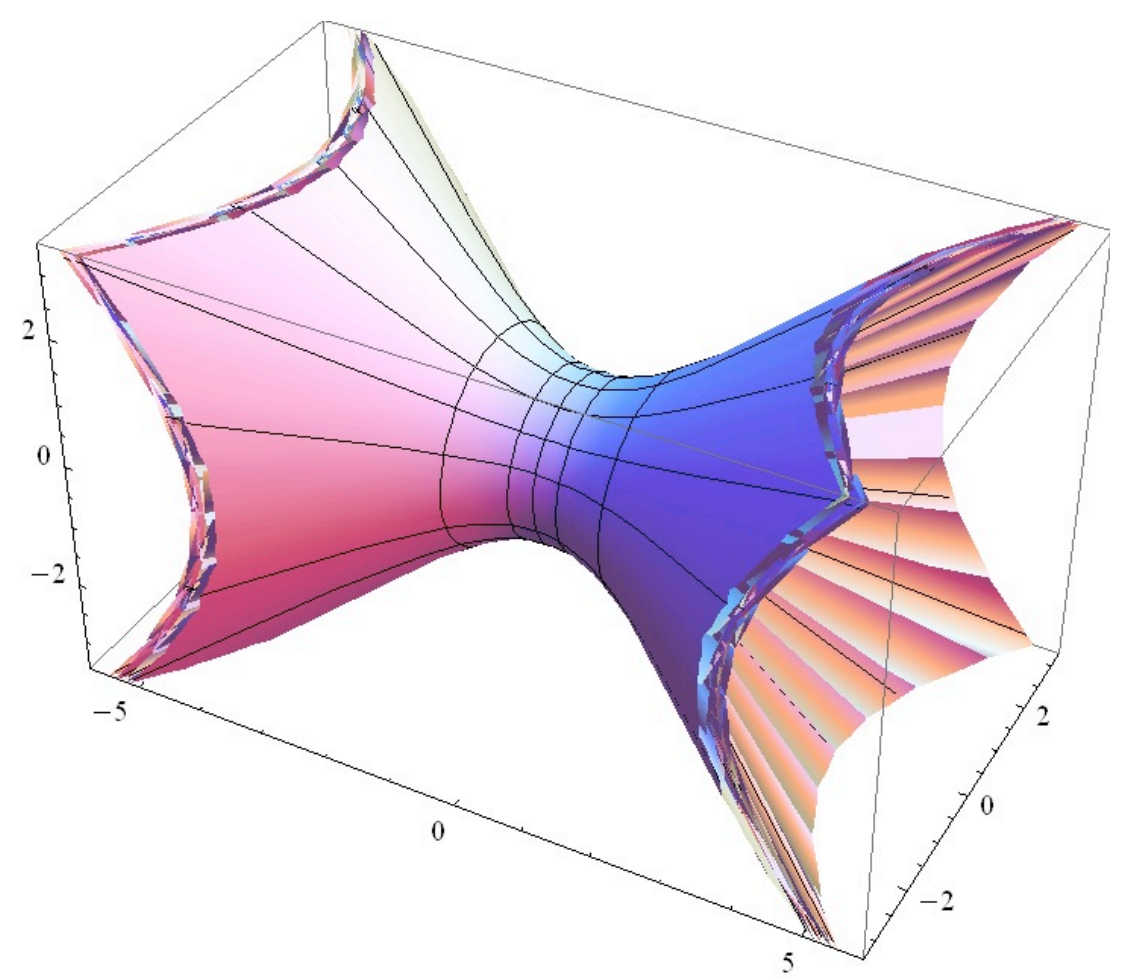

Figure 2: The harmonic evolute surface $h(s, t)$ with $s \in(-4,4)$ and $t \in(0,2 \pi)$

\section{References}

[1] Sipus, Z.M., Vladimir, V., The harmonic evolute of a surface in Minkowski 3-space, Mathematical Communications, 19, 43-55, 2014.

[2] Lopez, R., Sipus, Z.M., Gajcic, L.P., Protrka, I., Harmonic evolutes of B-scrolls with constant mean curvature in Lorentz-Minkowski space, International Journal of Geometric Methods in Modern Physics, 16 (5), 1950076, 2019.

[3] Körpinar, T., Kaymanli, G.U., On the harmonic evolute of quasi normal surfaces, Journal of Science and Arts, 1 (50), 55-64, 2020. 
[4] Eren, K., Kösal, H.H., Evolution of space curves and the special ruled surfaces with modified orthogonal frame, AIMS Mathematics, 5 (3), 2027-2039, 2020.

[5] Kelleci, A., Eren, K., On evolution of some associated type ruled surfaces, Mathematical Sciences and Applications E-Notes, 8 (2), 178-186, 2020.

[6] Hasimoto, H., Motion of a vortex filament and its relation to elastica, Journal of the Physical Society of Japan, 31, 293-294, 1971.

[7] Hasimoto, H., A soliton on a vortex filament, Journal of Fluid Mechanics, 51 (3), 477485, 1972.

[8] Rogers, C., Schief, W.K., Bäcklund and Darboux transformations, Cambridge University Press, 432, 2002.

[9] Abdel-All, N.H., Hussien, R.A., Youssef, T., Hasimoto surfaces, Life Science Journal, 9 (3), 556-560, 2012.

[10] Kelleci, A., Bektaş, M., Ergüt, M., The Hasimoto surface according to Bishop frame, Adiyaman University Journal of Science, 9 (1), 13-22, 2019.

[11] Erdoğdu, M., Özdemir, M., Geometry of Hasimoto surfaces in Minkowski 3-space, Mathematical Physics, Analysis and Geometry, 17, 169-181, 2014.

[12] Çakmak, A., Öklid 3-uzayında Hasimoto yüzeylerinin paralel yüzeyleri, Bitlis Eren Üniversitesi Fen Bilimleri Dergisi, 7 (1), 125-132, 2018. 